\title{
Análisis comparativo del transporte de carga minera en la Región Central
}

HenrRy Gastón CASAPia Soto ${ }^{1}$

\begin{abstract}
RESUMEN
La investigación muestra un análisis comparativo de infraestructura de transporte terrestre en el Perú, donde existe competencia intermodal, específicamente, entre la carretera y el ferrocarril en la Región Central. A partir de este análisis se establece qué medio de transporte de carga se utiliza para llevar mercancías como graneles sólidos, considerando a los productores y consumidores que compiten por la logística minera con destino al Puerto del Callao. Los resultados demuestran que al transferir la carga minera de la carretera al ferrocarril se reduce la congestión vehicular por el uso intensivo de camiones.
\end{abstract}

Palabras clave: infraestructura de transporte competencia intermodal; medios de transporte de carga; graneles sólidos; logística minera.

\section{INTRODUCCIÓN}

La Carretera Central ha colapsado por el incremento del tránsito debido, principalmente, a la excesiva presencia de camiones pesados; esto la vuelve peligrosa y ocasiona que los transportistas disminuyan su velocidad y asuman un mayor costo económico por la congestión vehicular debido al tiempo perdido en dicho tráfico, que en promedio es de $40 \mathrm{~km} / \mathrm{h}$ según Casapia (2014). Una solución a este problema es construir una nueva carretera; sin embargo, esto demandaría un proceso de alta ingeniería y de alto financiamiento, por lo que se necesita introducir una alternativa modal en el transporte terrestre de mercancías como los minerales. Por ello, esta investigación aborda a profundidad la contribución del ferrocarril, cuyo promedio de velocidad es de 25 $\mathrm{Km} / \mathrm{h}$ (con paradas en estaciones y sincronización de locomotoras y vagones), con la finalidad de atender los requerimientos de la actividad productiva nacional, y reducir la congestión de las carreteras producida por esta.

\section{Antecedentes}

Desde su aparición en el país, el transporte automotor por carretera se enfrentó competitivamente con el ferrocarril, el cual ya contaba con un desarrollo previo de 50 años. No obstante, el sistema de transporte ferroviario del centro y del sur del país viene presenciando un lento declive, a pesar de que actualmente en otros países se llega a una situación de equilibrio en cuanto a los volúmenes de carga que se transportan.

La productividad del Perú se robustece en la explotación minera por lo que ha habido una expansión de la red de carreteras con primacía sobre el transporte ferroviario; es así como el Ferrocarril Central compite especialmente en forma directa con la Carretera Central. Según Casapia (2014), estos dos modos de transporte competitivo comprenden la "bimodal central", la cual comunica las regiones de Pasco, Junín y Lima con el Puerto

1 Ingeniero civil por la Universidad Ricardo Palma con especialidad en Regulación de Concesiones de Infraestructura de Transporte (Lima, Perú). Actualmente, es consultor de Janus Consulting (Cusco, Perú)

ORCID: https://orcid.org/0000-0002-5776-6109

E-mail: janusconsulting.gg@gmail.com 
del Callao; de esto se deduce que la proporción de carga a través de camiones es casi cuatro veces la carga que se transporta en trenes, lo que conduce a la saturación en la carretera (ver Figura 1).
La Carretera Central, de 377 km de longitud, está concesionada a la empresa Deviandes S.A.C. desde el año 2010, la cual está encargada de realizar mantenimientos rutinarios y periódicos a fin de pos-

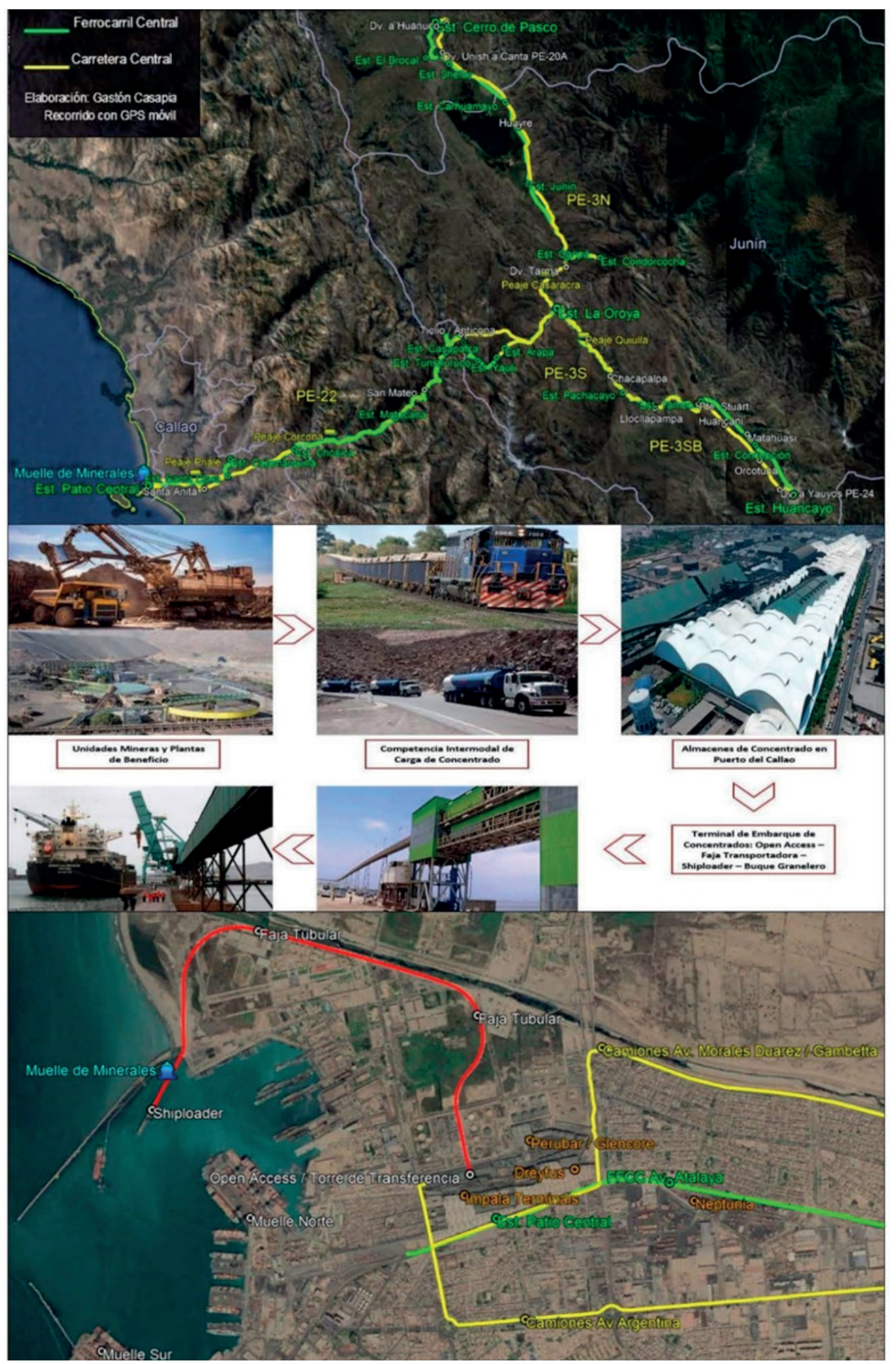

Figura 1. Flujo de la competencia intermodal de la bimodal central y embarque de concentrados en Puerto Callao.

Fuente: Elaboración propia con herramientas de orientación geoespacial. 
tergar su rehabilitación. Sus ingresos provienen del cobro de peajes en Corcona, Casaracra y Quiulla, al que se le agregaría un peaje adicional, amparados por ingresos mínimos anuales garantizados concedidos por el Estado, que para el año 2016 fueron de USD 14271590 sin incluir I.G.V. y por cada Unidad de Peaje, según se estipula en el Contrato de Concesión del Organismo Supervisor de la Inversión en Infraestructura de Transporte de Uso Público [Ositrán] (2010).

El Ferrocarril Central se concesionó en el año 1999 a Ferrovías Central Andina S.A., cuya infraestructura, material tractivo (locomotoras) y rodante (vagones y coches) pertenecieron a la Empresa Nacional de Ferrocarriles (Enafer). La vía férrea, con una trocha estándar de $1.44 \mathrm{~m}$ y una extensión de 477 km, viene siendo rehabilitada y su parque ferroviario, renovado. Sus tarifas proceden del cobro por uso de la vía por kilómetro recorrido, del cual se retribuye al Estado el $24.75 \%$ de los ingresos brutos (sin incluir ingresos del material tractivo o rodante, ni I.G.V) (Ositrán, 2006).

La importancia del almacenamiento y embarque de la carga proveniente de la Región Central se extiende hasta el Puerto del Callao, pues forma parte de la cadena logística de los minerales exportados entre la mina o la planta concentradora y los buques que se cargan en el puerto. Es así que desde finales del año 2014 la exportación se desarrolla a través de la concesión del Terminal de Embarque de Concentrados de Minerales a la empresa Transportadora Callao S.A., la cual posee un sistema hermético de faja transportadora que va desde un punto de acceso libre o público (open access) o torre de transferencia hacia el muelle (shiploader), previsto para atender 2000 toneladas por hora y naves de hasta 60000 toneladas de peso muerto (deadweight tonnage - DWT). Los beneficios de este sistema de embarque es que es 5 veces más rápido que el anterior, reduce 8 días de tiempo de espera en bahía, reduce 3 días de tiempo de estadía en muelle, y ha reemplazado aproximadamente 369000 viajes de camiones entre almacenes y el puerto, según Transportadora Callao (2018).

Esta investigación tiene como objetivo analizar la adecuada competencia intermodal del transporte de carga minera que disminuya la congestión vehicular de la cadena logística minera en la Región Central.

\section{HIPÓTESIS}

La adecuada competencia intermodal del transporte de carga ferroviaria disminuye la congestión vehicular de la cadena logística minera en la Región Central.

\section{JUSTIFICACIÓN}

El sector minero tradicional es el principal exportador del Perú. En el 2017, este sector generó alrededor del $61 \%$ del valor total de exportaciones FOB, que ascendió a USD 26809 millones, lo que representó un incremento del $27.4 \%$ en relación con el año anterior, debido al aumento de exportaciones de cobre $(36 \%)$, hierro $(27 \%)$, zinc $(32 \%)$, y otros minerales $(42 \%)$ (Superintendencia Nacional de Aduanas y de Administración Tributaria [SUNAT], 2018), lo que ha contribuido con el incremento del $\mathrm{PBI}$, que es el indicador que mide la capacidad de generación de riqueza en la economía del país.

Ante la ausencia de refinerías en el Perú, más del 95\% de la producción minera se envía al mercado exterior; dentro de esta se encuentran los concentrados de mineral, tales como cobre, plomo, plata, oro, zinc y molibdeno. Estos concentrados se han comercializado internacionalmente a través del canal privado, que está constituido por productores y comerciantes nacionales y extranjeros (traders), los cuales se encargan de movilizar actualmente el total de la producción exportable de dichos concentrados haciendo uso de la logística y sus medios de transporte.

Debido al uso extensivo de camiones de carga, con la premisa de que es el único medio para cumplir con los calendarios de comercialización, año tras año la congestión vehicular en la Carretera Central se incrementa en índices para lo que su infraestructura de transporte no fue diseñada.

La Región Central posee otro modo de transporte mucho más eficiente para grandes volúmenes de carga y largas distancias: el Ferrocarril Central; sin embargo, este no tiene las mismas condiciones de equidad, aun cuando el Plan Nacional de Desarrollo Ferroviario indica que su finalidad es que contribuya a la competitividad de las exportaciones (Resolución Ministerial N. ${ }^{\circ}$ 396-2016-MTC, 2016).

La infraestructura de transporte de uso público debe apuntar a que este llegue a más sitios y más rápido; a que sea más económico, más seguro, más cómodo; y principalmente que reduzca la brecha en infraestructura y que eleve la competitividad del país. En consecuencia, esta investigación analiza la correlación entre la problemática del exceso de camiones con carga minera en la Carretera Central, según la competencia intermodal, y su logística minera hacia puertos; así, se busca 
aminorar su circulación mediante el uso del ferrocarril, que es más eficiente y asequible.

\section{BASES TEÓRICAS}

\section{Competencia intermodal del transporte de carga}

El transporte de forma intermodal se da cuando se articulan diferentes modos de transporte, realizando el trasbordo de materiales y mercancías con una única medida de carga que podrían ser en contenedores $u$ otros elementos de transporte con capacidad de carga.

Tomando como punto de referencia el reparto modal del transporte de carga en diferentes economías del mundo -incluyendo 28 países de la Unión Europea, USA, Japón, China y Rusia-, los modos marítimo y terrestre en carretera trasladan el mayor porcentaje de carga con un 33\% cada uno, mientras que el transporte terrestre en ferrocarril se acerca con un $22 \%$ del total de carga en billones de toneladas-kilómetro, según la European Commission (2018). Sin embargo, esta competencia intermodal dista de nuestra realidad, en donde los estudios del Plan Intermodal de Transportes 20042023 establecen que existe un transporte de "carga interurbana" que se da en un $74 \%$ por la carretera, $13 \%$ por el modo ferroviario, $12 \%$ por el marítimo y $1 \%$ entre el modo fluvial y el aéreo (Ministerio de Transportes y Comunicaciones [MTC], 2005).

Según Rodrigue, Comtois y Slack (2013), "Los modos pueden competir o complementarse entre sí en términos de costo, velocidad, accesibilidad, frecuencia, seguridad, comodidad, etc." (p. 107) dependiendo de las siguientes condiciones: 1. Diferentes mercados geográficos, 2. Diferentes mercados del transporte, y 3. Diferentes niveles de servicio.

Si bien la existencia de la competencia intermodal depende de los mercados geográficos y del transporte, existen otros factores que afectan estos principios, tales como las condiciones de la infraestructura, las condiciones orográficas y los niveles de servicio en colapso o con congestión; por lo que se debe de optimizar la competencia intermodal del transporte de carga para largas distancias.

Según Thomson y Bull (2002), "la congestión es la condición que prevalece si la introducción de un vehículo en un flujo de tránsito aumenta el tiempo de circulación de los demás" (p. 110). En ese sentido, Ortúzar y Willumsen (2011) definen que la congestión surge cuando la intensidad de la demanda se aproxima a la capacidad de la instalación (infraestructura de carretera o ferrocarril) y el tiempo requerido para utilizarla sobrepasa la media establecida de baja demanda.

\section{Cadena logística de la comercialización minera}

La cadena de valor en la industria minera - desde la extracción en minas, el procesamiento y la comercialización de concentrados hasta su exportación en puertos- determina la eficiencia de su logística minera; sin embargo, el reajuste del costo logístico depende de los operadores según su capacidad de reducir sus costos fijos y el alto índice de especialización logística, por lo que los empresarios mineros deben de tomar la decisión de seleccionarlos por criterios de comunicación, valor del flete, capacidad de atención, y calidad de entrega (Galo, Ribeiro, Mergulhão, y Vieira, 2018).

\section{METODOLOGÍA}

Esta investigación no experimental se realiza en base a un encuadre metodológico descriptivo-comparativo, estipulado en un análisis comparativo de la demanda del transporte de carga minera terrestre tanto en la Carretera Central como en el Ferrocarril Central en servicio, para conocer la alternativa modal al tráfico existente en la infraestructura vehicular.

Los modos de infraestructura terrestre de uso público en la Región Central son únicos, por lo que la población y tamaño del estudio son los mismos; estos son el Ferrocarril del Centro y la Carretera IIRSA Centro Tramo 2.

Se evalúa la cadena logística minera que comprende la competencia intermodal de la carga, es decir, desde las plantas en las unidades mineras hasta el Puerto del Callao, analizando (1) el Índice Medio Diario Anual (IMDA) de los vehículos que pasan por las estaciones de conteo de la Carretera Central y sus rutas alternas y (2) la demanda de trenes según los reportes de envíos del Ferrocarril Central. Así, se tiene el total de unidades que llegan a almacenamiento en el puerto, las mismas que proceden luego a despachar la carga minera por medios marítimos a través del movimiento de tráfico del Terminal de Embarque de Concentrados de Minerales en el Puerto del Callao.

\section{RESULTADOS}

\section{Productividad minera en la Región Central}

El área de influencia de la investigación abarca la Región Central del país, en la cual ha habido actividad de producción minera metálica por muchos años, particularmente en la orografía andina de las 
Regiones de Lima, Junín y Pasco. En consecuencia, la investigación se circunscribe directamente en 20 Unidades Mineras, en 15 de las cuales de aplica el método de explotación de minería subterránea; en 4, de minería superficial; y en 1, la de Cajamarquilla, de refinería (Ministerio de Energía y Minas [MINEM], 2018) (ver Tabla 1).

\section{Competencia de la bimodal central en transpor- te de carga minera}

Hace aproximadamente un siglo, el traslado minero desde las unidades de la empresa Cerro de Pasco Copper Corporation hacia La Oroya y El Callao se realizaba por medio del ferrocarril; sin embargo, la aparición posterior de los vehículos de carga pesados y la culminación de la Carretera Central en 1936 hicieron ver que su logística podría ser reducida, sobre todo porque no existía en aquel momento, ni existe hoy en día, un justiprecio entre el pago de peaje por eje y el deterioro por eje equivalente que ocasiona el sobrecargar la vía.

Debido a la dificultad para obtener información sobre el transporte de minerales a través de las carreteras del país, se realiza el conteo de las unidades de transporte terrestre al atravesar puntos de control o estaciones. De esta forma, el Ministerio de

Tabla 1. Unidades mineras de la Región Central.

\begin{tabular}{|c|c|c|c|c|c|c|c|}
\hline Id & $\begin{array}{l}\text { Mét. } \\
\text { Expl. }\end{array}$ & Titular & Unidad & Reg. & Prov. & Dist. & Prod. \\
\hline 1 & $\begin{array}{l}\text { Minería } \\
\text { Subte. }\end{array}$ & Comp. Minera Argentum S.A. & Anticona & Junín & Yauli & Yauli & $\mathrm{Zn}, \mathrm{Ag}, \mathrm{Cu}, \mathrm{Pb}$ \\
\hline 2 & $\begin{array}{l}\text { Minería } \\
\text { Subte. }\end{array}$ & Comp. Minera Argentum S.A. & Manuelita & Junín & Yauli & Yauli & $\mathrm{Pb}, \mathrm{Zn}, \mathrm{Ag}, \mathrm{Cu}$ \\
\hline 3 & $\begin{array}{l}\text { Minería } \\
\text { Subte. }\end{array}$ & Comp. Minera Argentum S.A. & Morococha & Junín & Yauli & Morococha & $\mathrm{Pb}, \mathrm{Zn}, \mathrm{Ag}, \mathrm{Cu}$ \\
\hline 4 & $\begin{array}{l}\text { Minería } \\
\text { Subte. }\end{array}$ & $\begin{array}{c}\text { Nexa Resources Atacocha } \\
\text { S.A.A. }\end{array}$ & Atacocha & Pasco & Pasco & $\begin{array}{l}\text { San Fco. Asís } \\
\text { de Yarusyacán }\end{array}$ & $\begin{array}{c}\mathrm{Pb}, \mathrm{Zn}, \mathrm{Au}, \mathrm{Ag} \\
\mathrm{Cu}, \mathrm{Bi}\end{array}$ \\
\hline 5 & $\begin{array}{l}\text { Minería } \\
\text { Subte. }\end{array}$ & Comp. Minera Casapalca S.A. & Americana & Junín & Yauli & Yauli & $\mathrm{Zn}, \mathrm{Ag}, \mathrm{Cu}, \mathrm{Pb}$ \\
\hline 6 & $\begin{array}{l}\text { Minería } \\
\text { Subte. }\end{array}$ & $\begin{array}{l}\text { Comp. Minera San Ignacio de } \\
\text { Morococha S.A.A. }\end{array}$ & Palmapata & Junín & Chancha-mayo & San Ramón & $\mathrm{Pb}, \mathrm{Zn}$ \\
\hline 7 & $\begin{array}{l}\text { Minería } \\
\text { Subte. }\end{array}$ & $\begin{array}{c}\text { Comp. Minera San Ignacio de } \\
\text { Morococha S.A.A. }\end{array}$ & San Vicente & Junín & Chancha-mayo & Vitoc & $\mathrm{Pb}, \mathrm{Zn}$ \\
\hline 8 & $\begin{array}{l}\text { Minería } \\
\text { Super. }\end{array}$ & $\begin{array}{c}\text { Emp. Administradora Cerro } \\
\text { S.A.C. }\end{array}$ & Cerro de Pasco & Pasco & Pasco & Simón Bolívar & $\mathrm{Cu}, \mathrm{Pb}, \mathrm{Zn}, \mathrm{Au}, \mathrm{Ag}$ \\
\hline 9 & $\begin{array}{l}\text { Minería } \\
\text { Subte. }\end{array}$ & $\begin{array}{c}\text { Emp. Minera Los Quenuales } \\
\text { S.A. }\end{array}$ & Yauliyacu & Lima & Huarochirí & Chicla & $\mathrm{Zn}, \mathrm{Ag}, \mathrm{Pb}, \mathrm{Cu}$ \\
\hline 10 & $\begin{array}{l}\text { Minería } \\
\text { Subte. }\end{array}$ & $\begin{array}{c}\text { Nexa Resources El Porvenir } \\
\text { S.A.C. }\end{array}$ & Milpo No1 & Pasco & Pasco & Yanacancha & $\mathrm{Cu}, \mathrm{Pb}, \mathrm{Zn}, \mathrm{Au}, \mathrm{Ag}$ \\
\hline 11 & $\begin{array}{l}\text { Minería } \\
\text { Super. }\end{array}$ & Minera Chinalco Perú S.A. & Toromocho & Junín & Yauli & Morococha & $\begin{array}{c}\mathrm{Cu}, \mathrm{Mo}, \mathrm{Zn}, \mathrm{Ag}, \\
\text { As }\end{array}$ \\
\hline 12 & $\begin{array}{l}\text { Minería } \\
\text { Subte. }\end{array}$ & $\begin{array}{c}\text { Soc. Minera Austria Duvaz } \\
\text { S.A.C. }\end{array}$ & Austria Duvaz & Junín & Yauli & Morococha & $\mathrm{Pb}, \mathrm{Zn}, \mathrm{Ag}, \mathrm{Cu}$ \\
\hline 13 & $\begin{array}{l}\text { Minería } \\
\text { Super. }\end{array}$ & Soc. Minera El Brocal S.A.A. & Colquijirca N¹ & Pasco & Pasco & Simón Bolívar & $\mathrm{Cu}, \mathrm{Au}, \mathrm{Ag}, \mathrm{As}$ \\
\hline 14 & $\begin{array}{l}\text { Minería } \\
\text { Super. }\end{array}$ & Soc. Minera El Brocal S.A.A. & Colquijirca $\mathrm{N}^{\circ} 2$ & Pasco & Pasco & Tinyahuarco & $\mathrm{Zn}, \mathrm{Ag}, \mathrm{Pb}, \mathrm{Cu}$ \\
\hline 15 & $\begin{array}{l}\text { Minería } \\
\text { Subte. }\end{array}$ & Volcan Comp. Minera S.A.A. & Andaychagua & Junín & Yauli & Huay-Huay & $\mathrm{Zn}, \mathrm{Ag}, \mathrm{Pb}, \mathrm{Cu}$ \\
\hline 16 & $\begin{array}{l}\text { Minería } \\
\text { Subte. }\end{array}$ & Volcan Comp. Minera S.A.A. & Carahuacra & Junín & Yauli & Yauli & $\mathrm{Zn}, \mathrm{Ag}, \mathrm{Cu}, \mathrm{Pb}$ \\
\hline 17 & $\begin{array}{l}\text { Minería } \\
\text { Subte. }\end{array}$ & Volcan Comp. Minera S.A.A. & Morada & Junín & Yauli & Yauli & $\mathrm{Cu}, \mathrm{Pb}, \mathrm{Zn}, \mathrm{Ag}$ \\
\hline 18 & $\begin{array}{l}\text { Minería } \\
\text { Subte. }\end{array}$ & Volcan Comp. Minera S.A.A. & San Cristóbal & Junín & Yauli & Yauli & $\mathrm{Cu}, \mathrm{Pb}, \mathrm{Zn}, \mathrm{Ag}$ \\
\hline 19 & $\begin{array}{l}\text { Minería } \\
\text { Subte. }\end{array}$ & Volcan Comp. Minera S.A.A. & Ticlio & Junín & Yauli & Morococha & $\mathrm{Cu}, \mathrm{Pb}, \mathrm{Zn}, \mathrm{Ag}$ \\
\hline 20 & Refinería & $\begin{array}{c}\text { Nexa Resources Cajamarquilla } \\
\text { S.A. }\end{array}$ & $\begin{array}{c}\text { Refinería de } \\
\text { Cajamarquilla }\end{array}$ & Lima & Lima & Lurigancho & $\mathrm{Cd}, \mathrm{Cu}, \mathrm{Zn}, \mathrm{Ag}$ \\
\hline
\end{tabular}

Fuente: Elaboración propia a partir del Mapa de Principales Unidades Mineras en Producción (MINEM, 2018). 
Transportes y Comunicaciones consigue el IMDA por tipo de vehículo según tramos viales. Para el ámbito de la Región Central, se toman en cuenta los datos del MTC (MTC, 2013 y MTC, 2016) considerando que el 2016 fue el último año en que se publicó información de dicho ministerio- y se analizan la Tabla 2 y la Figura 2.

En la Tabla 2 se aprecia el número de vehículos que hacen uso de la Carretera Central (PE-22, PE-3N y PE-3S) y las rutas alternas en ambos sentidos. Según la clasificación por demanda del Manual de Carreteras del MTC (Resolución Directoral $N^{\circ}$ 03-2018-MTC, 2018), una vía con un IMDA mayor a $6000 \mathrm{veh} / \mathrm{día}$, con dos o más carriles de $3.60 \mathrm{~m}$ de ancho mínimo y un separador central de 6.00 $\mathrm{m}$ sería una Autopista de Primera Clase; es decir que su sección típica mediría un ancho mínimo de $20.40 \mathrm{~m}$. Sin embargo, la Carretera Central aún posee una calzada de $6.60 \mathrm{~m}$, razón por la cual aún presenta inconvenientes con su diseño geométrico y, por lo tanto, problemas de congestión vehicular.

Es conveniente observar cómo el flujo de los medios de transporte pesado de seis ejes —como los camiones T3S3 (tractor de tres ejes y semitrailer de tres ejes) y los encapsulados mineros con que se trasladan los concentrados hacia puerto- han ido aumentando a razón de 1 a $5 \%$ anual en promedio, aunque en Carhuamayo (entre Cerro de Pasco y La Oroya) este aumento llegó hasta $20 \%$, mientras que en otras rutas como la PE-18 en Churín (entre Huaura y Ambo) el incremento fue del $43 \%$ y en la PE-20A en Yangas (entre Lima y Unish) se llegó incluso a un $108 \%$ anual.

Además de analizar el traslado de concentrados de minerales en vehículos pesados, también se puede comparar el movimiento de trenes (locomotoras y vagones) y sus respectivas cargas a través del Ferrocarril Central, cuya información proviene del concesionario Ferrovías Central Andina S.A. (FVCA); así, se tiene la Tabla 3 del transporte de concentrados de minerales (Ferrovías Central Andina [FVCA], 2017).

Una vez llegado el concentrado de zinc al Callao, un total de $10037 \mathrm{TMH}$ fueron trasladados a la Refinería de Cajamarquilla por Nexa Resources Cajamarquilla S.A. para obtener barras de zinc.

Tabla 2. IMDA 2013 - 2016 en la Región Central.

\begin{tabular}{|c|c|c|c|c|c|c|c|c|c|c|c|}
\hline \multirow{2}{*}{$\begin{array}{l}\text { Estación de } \\
\text { Control }\end{array}$} & \multirow{2}{*}{$\begin{array}{c}\text { Ruta } \\
\text { (ambos sentidos) }\end{array}$} & \multicolumn{5}{|c|}{2013} & \multicolumn{5}{|c|}{2016} \\
\hline & & V.L. & T.P. & T.C. & IMDA & T3S3 & V.L. & T.P. & T.C. & IMDA & T3S3 \\
\hline \multirow{2}{*}{ Corcona } & \multirow{2}{*}{ PE-22 } & 2150 & 1034 & 2376 & 5560 & 1061 & 2570 & 1090 & 2539 & 6199 & 1077 \\
\hline & & $39 \%$ & $19 \%$ & $43 \%$ & & $45 \%$ & $41 \%$ & $18 \%$ & $41 \%$ & & $42 \%$ \\
\hline \multirow{2}{*}{ Tambo de Viso } & \multirow{2}{*}{ PE-22 } & 2121 & 750 & 2558 & 5429 & 1081 & 2370 & 876 & 2764 & 6010 & 1182 \\
\hline & & $39 \%$ & $14 \%$ & $47 \%$ & & $42 \%$ & $39 \%$ & $15 \%$ & $46 \%$ & & $43 \%$ \\
\hline \multirow{2}{*}{ Casaracra } & \multirow{2}{*}{ PE-3N } & 1272 & 446 & 1730 & 3448 & 688 & 1776 & 654 & 2112 & 4542 & 795 \\
\hline & & $37 \%$ & $13 \%$ & $50 \%$ & & $40 \%$ & $39 \%$ & $14 \%$ & $46 \%$ & & $38 \%$ \\
\hline \multirow{2}{*}{ Carhuamayo } & \multirow{2}{*}{ PE-3N } & 1054 & 302 & 1017 & 2373 & 490 & 1223 & 407 & 1717 & 3347 & 788 \\
\hline & & $44 \%$ & $13 \%$ & $43 \%$ & & $48 \%$ & $37 \%$ & $12 \%$ & $51 \%$ & & $46 \%$ \\
\hline \multirow{2}{*}{ Quiulla } & \multirow{2}{*}{ PE-3S } & 1520 & 517 & 884 & 2921 & 464 & 2723 & 617 & 984 & 4324 & 352 \\
\hline & & $52 \%$ & $18 \%$ & $30 \%$ & & $52 \%$ & $63 \%$ & $14 \%$ & $23 \%$ & & $36 \%$ \\
\hline \multirow{2}{*}{ Churín } & \multirow{2}{*}{ PE-18 } & 219 & 63 & 130 & 412 & 35 & 585 & 196 & 245 & 1026 & 80 \\
\hline & & $7 \%$ & $2 \%$ & $4 \%$ & & $27 \%$ & $14 \%$ & $5 \%$ & $6 \%$ & & $33 \%$ \\
\hline \multirow{2}{*}{ Yangas } & \multirow{2}{*}{ PE-20A } & 480 & 209 & 66 & 755 & 8 & 737 & 306 & 173 & 1216 & 34 \\
\hline & & $16 \%$ & $7 \%$ & $2 \%$ & & $12 \%$ & $17 \%$ & $7 \%$ & $4 \%$ & & $20 \%$ \\
\hline \multirow{2}{*}{ Pirca } & \multirow{2}{*}{ PE-1NC } & 217 & 35 & 26 & 278 & 0 & 282 & 50 & 32 & 364 & 0 \\
\hline & & $7 \%$ & $1 \%$ & $1 \%$ & & $0 \%$ & $7 \%$ & $1 \%$ & $1 \%$ & & $0 \%$ \\
\hline \multirow{2}{*}{ Pacarán } & \multirow{2}{*}{ PE-24 } & 220 & 141 & 67 & 428 & 0 & 324 & 235 & 80 & 639 & 2 \\
\hline & & $8 \%$ & $5 \%$ & $2 \%$ & & $0 \%$ & $7 \%$ & $5 \%$ & $2 \%$ & & $3 \%$ \\
\hline
\end{tabular}

Nota: V.L.: Vehículo Ligero (autos y camionetas).

T.P.: Transporte Público (camioneta rural, micros y ómnibus).

T.C.: Transporte de Carga (camiones 2 y 3 ejes, articulados).

Fuente: Elaboración propia a partir de IMDA por tipo de vehículo, según tramos viales (MTC, 2013; MTC, 2016). 


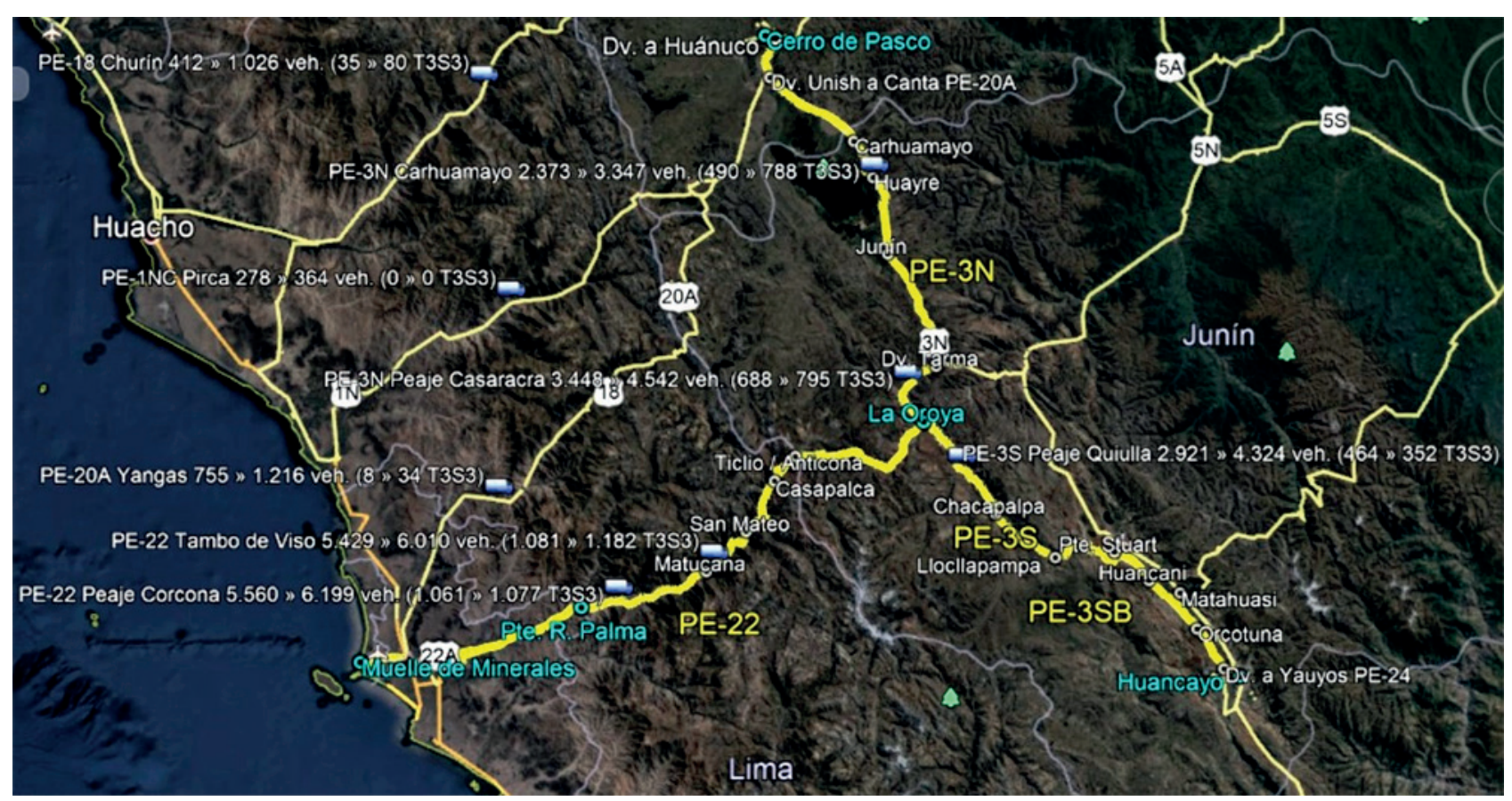

Figura 2. IMDA 2013 - 2016 en la Región Central

Fuente: Elaboración propia en Google Earth, a partir del IMDA, por tipo de vehículo, según tramos viales (MTC, 2013; MTC, 2016).

Tabla 3. Transporte de carga en Ferrocarril Central, destino Callao 2017.

\begin{tabular}{|c|c|c|c|c|}
\hline \multicolumn{5}{|c|}{ Concentrado de Cu de U.M./planta a Puerto del Callao } \\
\hline Empresa & Origen & Destino & TMH & $\%$ \\
\hline Minera Chinalco Perú S.A. & Tunshuruco & Patio Central & 1169924 & $99 \%$ \\
\hline Sociedad Minera El Brocal S.A.A. & El Brocal & Patio Central & 11374 & $1 \%$ \\
\hline Milpo Andina Perú S.A.C. & Unish & Patio Central & 1264 & $0 \%$ \\
\hline Compañía Minera Atacocha S.A.A. & Unish & Patio Central & 158 & $0 \%$ \\
\hline & & & 1182719 & \\
\hline \multicolumn{5}{|c|}{ Concentrado de $\mathrm{Pb}$ de U.M./planta a Puerto del Callao } \\
\hline Empresa & Origen & Destino & TMH & $\%$ \\
\hline Milpo Andina Perú S.A.C. & Unish & Patio Central & 15443 & $51 \%$ \\
\hline Compañía Minera Atacocha S.A.A. & Unish & Patio Central & 14108 & $46 \%$ \\
\hline Volcan Compañía Minera S.A.A. & Mahr Túnel & Patio Central & 835 & $3 \%$ \\
\hline & & & 30385 & \\
\hline \multicolumn{5}{|c|}{ Concentrado de Zn de U.M./planta a Puerto del Callao } \\
\hline Empresa & Origen & Destino & TMH & $\%$ \\
\hline Volcan Compañía Minera S.A.A. & Carahuacra / Mahr Túnel & Patio Central & 173562 & $92 \%$ \\
\hline Milpo Andina Perú S.A.C. & Unish & Patio Central & 9115 & $5 \%$ \\
\hline Sociedad Minera El Brocal S.A.A. & El Brocal & Patio Central & 4131 & $2 \%$ \\
\hline Empresa Administradora Cerro S.A.C. & Cerro de Pasco & Patio Central & 1607 & $1 \%$ \\
\hline Empresa Minera Los Quenuales S.A. & Casapalca & Patio Central & 161 & $0 \%$ \\
\hline & & & 188577 & \\
\hline
\end{tabular}

Fuente: Elaboración propia a partir del Reporte Acumulado de Clientes por Carga (FVCA, 2017). 
En el 2017, el origen del transporte de concentrado de cobre, plomo y zinc a través de la línea férrea con destino el Puerto del Callao (Patio Central) fueron las plantas de beneficio y/o unidades mineras en producción. Los principales clientes del ferrocarril según concentrado fueron la Minera Chinalco con el $99 \%$ del cobre; Volcan con el $92 \%$ del zinc; $y$, en el caso del plomo, Milpo con el $51 \%$ y Atacocha con el $46 \%$; estas dos últimas han pasado a conformar actualmente la empresa Nexa Resources, que en consecuencia se llevaría el $97 \%$ del plomo.

\section{Logística minera en el Puerto del Callao}

Independientemente de la cadena de suministros hacia las minas, la cadena logística organiza el transporte para entregar los productos procedentes de las plantas de beneficio hacia el cliente final. Ya sea utilizando la bimodal central u otras rutas alternativas, el propósito de las empresas mineras es completar dicha cadena hacia una refinería o el Puerto del Callao, según el contrato de comercialización que haya suscrito.

Debido a las exigencias con respecto a las entregas de minerales, la mayoría de mineras subcontratan la organización del transporte a operadores logísticos o traders, los cuales ofrecen el servicio de asesoría logística minera, que consiste en contratar a las empresas de transporte, recoger y trasladar la mercadería, contratar depósitos para el almacenamiento, lidiar con trámites aduaneros y envíos marítimos, etc. Entre estos operadores logísticos, estratégicamente se encuentran Trafigura Perú S.A.C. (ex Cormín) y Glencore Perú S.A.C.

En el Plan de Negocios del Terminal de Embarque de Concentrados (Transportadora Callao, 2018) se detalla el tráfico del año 2017 de los almacenes de alimentación utilizados en puerto, tales como Impala Terminals con el $45 \%$, Perubar con el $33 \%$, Chinalco con el $15 \%$, y Dreyfus con el $7 \%$. Esto se complementa con la información del Resumen General de Naves y Tráfico de Carga en el Terminal de Embarque de Concentrados de Minerales (Ositrán, 2018) de la Tabla 4.

Se atendieron 182 naves o bulk carriers con un promedio de 16336 TM, que sumó un total de 2973 090 toneladas de carga para el año 2017, lo que representó el $77 \%$ del total exportado de concentrados de mineral en el Puerto del Callao y el $26 \%$ del total exportado en el Perú.

La principal problemática que se aprecia en el Puerto del Callao es que aún parte de los concentrados

Tabla 4. Exportación de concentrados de Transportadora Callao 2017.

\begin{tabular}{|c|c|c|c|c|c|}
\hline Mes & $\begin{array}{l}\text { Total de } \\
\text { naves }\end{array}$ & $\begin{array}{c}\text { Granel sólido - Mineral } \\
\text { Zn TM }\end{array}$ & $\begin{array}{c}\text { Granel sólido - Mineral } \\
\text { Cu TM }\end{array}$ & $\begin{array}{c}\text { Granel sólido - Mineral } \\
\mathrm{Pb} \text { TM }\end{array}$ & $\begin{array}{c}\text { Total Exportaciones } \\
\text { TM }\end{array}$ \\
\hline Enero & 17 & 79596 & 132853 & & 212449 \\
\hline Febrero & 18 & 98092 & 163638 & 48239 & 309968 \\
\hline Marzo & 14 & 90545 & 81275 & 14044 & 185865 \\
\hline Abril & 12 & 60495 & 133774 & 19448 & 213718 \\
\hline Mayo & 14 & 138558 & 146879 & 25241 & 310678 \\
\hline Junio & 20 & 117725 & 123643 & 33404 & 274772 \\
\hline Julio & 12 & 80737 & 85057 & 29781 & 195576 \\
\hline Agosto & 11 & 54873 & 116683 & 14937 & 186494 \\
\hline Septiembre & 17 & 126418 & 190910 & 16324 & 333652 \\
\hline Octubre & 13 & 82877 & 92490 & 29045 & 204412 \\
\hline Noviembre & 16 & 104787 & 127109 & 19495 & 251391 \\
\hline Diciembre & 18 & 141159 & 112332 & 40625 & 294116 \\
\hline \multirow[t]{2}{*}{ Anual } & 182 & 1175862 & 1506644 & 290584 & 2973090 \\
\hline & & $40 \%$ & $51 \%$ & $10 \%$ & $100 \%$ \\
\hline \multicolumn{4}{|c|}{ Total TMH exportado por Transportadora Callao } & 2973090 & $77 \%$ \\
\hline \multicolumn{4}{|c|}{ Total TMH exportado en big bags en contenedor } & 880385 & $23 \%$ \\
\hline \multicolumn{4}{|c|}{ Total TMH exportado por Puerto del Callao } & 3853475 & \\
\hline
\end{tabular}

Fuente: Elaboración propia a partir de Resumen General de Naves y Tráfico de Carga en Terminal de Embarque de Concentrados de Minerales (Ositrán, 2018). 
se exportan en big bags dentro de contenedores, lo que equivale a un $23 \%$ del total de exportaciones. Por esta razón, el concesionario ha venido registrando pérdidas en sus estados de resultados, ya que la carga atendida por el Terminal es sustancialmente menor a la proyectada.

Con la información recolectada sobre las unidades mineras de la Región Central, y sus plantas concentradoras, se puede estimar la cantidad de camiones pesados que se desplazan por esta región, el número de vagones transportadores de concentrado de mineral y las exportaciones que hacen uso de la faja transportadora del Terminal de Embarque de Concentrados de Minerales del Callao.

\section{DISCUSIÓN}

En el 2017, Transportadora Callao movilizó un total de $2973090 \mathrm{TMH}$ de concentrados de cobre, zinc y plomo; si se considera que el mismo año el Ferrocarril Central transportó al puerto $1391644 \mathrm{TMH}$ de concentrados con las mismas características, se puede deducir que la diferencia de estos montos son las toneladas que fueron enviadas en camiones al Callao, esto es, $1581446 \mathrm{TMH}$; es decir, del total transportado, un $47 \%$ se movilizó en vagones del ferrocarril y un 53\% por medio de camiones.

Dado que no existe mayor investigación al respecto, salvo el comparativo de la bimodal central, y con base en lo expuesto, se sustenta que la mayoría de mineras subcontratan a traders que prefieren el uso de empresas de transporte en camiones.

Si se considera que un camión encapsulado de concentrado minero posee una carga útil promedio de $30 \mathrm{TMH}$, la cantidad de camiones por año que llegaron a puerto fue de 52715 , con una frecuencia de 144 camiones/día.

\section{PRUEBA DE HIPÓTESIS}

Como se puede apreciar en la Tabla 2, el IMDA en el punto de control de Corcona fue de 6199 vehículos; si se transfiere la carga de la carretera al ferrocarril, retirando tan solo un promedio de 288 camiones en ambos sentidos, el IMDA se reduciría en casi el 5\%. Al incluir en el cambio de carga a los camiones de transporte de big bags de la Tabla 4, se reemplazarían por día alrededor de 448 unidades, es decir, el $42 \%$ de camiones de 6 ejes, con lo que se obtendría la descongestión de vehículos pesados no solo en la carretera sino particularmente en el acceso a Lima y al puerto marítimo del Callao. Así, el análisis demuestra que la adecuada competencia intermodal del transporte de carga ferroviaria disminuye la congestión vehicular de la cadena logística minera.

\section{CONCLUSIONES Y RECOMENDACIONES}

El desarrollo de infraestructura con respecto al modo de transporte terrestre ha venido consolidándose según el protagonismo tecnológico de sus medios de transporte. Es así que los costos promedio de operación de un camión -combustible, mantenimiento, repuestos y seguros- son significativamente menores que el material tractivo y rodante que utiliza un tren; asimismo, la infraestructura de transporte en carreteras con un pavimento flexible tiene un costo menor en inversión que la vía férrea (Casapia, 2014); no obstante, el primero recibe un mayor subsidio, a pesar de que el ferrocarril posee mayores costos hundidos, con un periodo de uso en promedio de 80 años en operación antes de ser rehabilitados.

Tras la discusión, se tiene que el modo ferroviario debería garantizar el beneficio socioeconómico del país, reduciendo la congestión vehicular, ahorrando el tiempo de traslado, el gasto de operación vehicular, el decrecimiento de accidentes de tránsito y, especialmente, disminuyendo los costos derivados del uso intensivo de camiones; es decir, debería primar una política de seguridad, de salud medio ambiental y de responsabilidad social.

El desarrollo de la infraestructura de transporte es necesaria para reducir los costos logísticos e incrementar el crecimiento sostenible de la región, de modo que se cierre la brecha de infraestructura que en nuestro país sería una buena opción para lograr la solidez del Plan Nacional de Infraestructura para la Competitividad (D. S. N. ${ }^{\circ}$ 238-2019-EF, 2019), cuya brecha a corto plazo en transporte ferroviario considera S/20 430000 000, mediante la conectividad multimodal, integrando la productividad y sus regiones utilizando los modos de la carretera, del ferrocarril y del acuático.

\section{REFERENCIAS BIBLIOGRÁFICAS}

[1] Casapia, G. (2014). Carretera o Ferrocarril ¿Qué modo de transporte usar? Lima, Perú: Universidad Ricardo Palma.

[2] D. S. N. ${ }^{\circ}$ 238-2019-EF. Plan Nacional de Infraestructura para la Competitividad. Diario Oficial El Peruano (28 de julio del 2019).

[3] European Commission. (2018). EU Transport Statistical Pocketbook. Luxembourg, 
Publications Office of the European Union. Recuperado de https://doi.org/10.2832/491038

[4] Ferrovías Central Andina - FVCA (2017). Reporte Acumulado de Clientes por Carga. Lima, Perú.

[5] Galo, N., Ribeiro, P., Mergulhão, R., y Vieira, J. (2018). Selección de proveedor de servicios logísticos: alineación entre criterios e indicadores. Innovar, 28(69), 55-67. Recuperado de https://doi.org/10.15446/ innovar.v28n69.71696

[6] Ministerio de Energía y Minas. (2018). Mapa de las Principales Unidades Mineras en Producción 2018. Lima, Perú.

[7] Ministerio de Transportes y Comunicaciones. (2005). Plan Intermodal de Transportes PIT 2004-2023. Lima, Perú.

[8] Ministerio de Transportes y Comunicaciones. (2013). Índice Medio Diario Anual - IMDA. Lima, Perú.

[9] Ministerio de Transportes y Comunicaciones (2016). Índice Medio Diario Anual - IMDA. Lima, Perú.

[10] Organismo Supervisor de la Inversión en Infraestructura de Transporte de Uso Público. (2006). Contrato de Concesión del Ferrocarril del Centro. Lima, Perú.

[11] Organismo Supervisor de la Inversión en Infraestructura de Transporte de Uso Público. (2010). Contrato de Concesión del IIRSA Centro Tramo 2: Puente Ricardo Palma - La Oroya - Huancayo y La Oroya - Dv. Cerro de Pasco. Lima, Perú.
[12] Organismo Supervisor de la Inversión en Infraestructura de Transporte de Uso Público. (2018). Resumen General de Naves y Tráfico de Carga en el Terminal de Embarque de Concentrados de Minerales 2017. Lima, Perú.

[13] Ortúzar, J., y Willumsen, L. (2011). Modelling Transport. Chichester, UK: Wiley Publication. Recuperado de https://doi. org/10.1002/9781119993308

[14] Resolución Ministerial N..$^{\circ}$ 396-2016-MTC. Aprueban el "Plan Nacional de Desarrollo Ferroviario". Diario Oficial El Peruano (16 de junio del 2016).

[15] Resolución Directoral $\quad N^{\circ}$ 03-2018-MTC, Aprueban Manual de Carreteras: Diseño Geométrico DG 2018. Diario Oficial El Peruano (07 de febrero del 2018).

[16] Rodrigue, J., Comtois, C., y Slack, B. (2013). The Geography of Transport Systems. Abingdon, UK: Routledge, Taylor \& Francis Group. Recuperado de https://doi. org/10.4324/9780203371183

[17] Superintendencia Nacional de Aduanas y de Administración Tributaria (2018). Anuario Estadístico de Comercio Exterior 2017. Lima, Perú.

[18] Thomson, I., y Bull, A. (2002). La Congestión del Tránsito Urbano: Causas y Consecuencias Económicas y Sociales. Revista de la CEPAL, (76), 109-121. Recuperado de http://hdl. handle.net/11362/10804

[19] Transportadora Callao (2018). Plan de Negocios del Terminal de Embarque de Concentrados de Minerales. Lima, Perú. 


\section{Comparative Analysis of Mining Freight in the Central Region}

HenrRy Gastón CASAPIA Soto ${ }^{1}$

\begin{abstract}
This study provides a comparative analysis of the land transportation infrastructure in Peru, where there is intermodal competition, specifically between road and rail in the Región Central (Central Region). Based on this analysis, which means of freight transport used to carry goods such as solid bulk, considering producers and consumers competing for mining logistic contracts to the Port of Callao, is established. The results show that transferring mining freight from road to rail reduces vehicular congestion caused by the intensive use of trucking.
\end{abstract}

Keywords: Transportation infrastructure; intermodal competition; freight transportation; solid bulks; mining logistics.

\section{INTRODUCTION}

The Carretera Central has collapsed due to the increase in traffic, principally due to the excessive presence of heavy trucks that make the highway dangerous and cause drivers to reduce their speed and assume higher economic impacts due to the time lost in said traffic caused by vehicle congestion, which averages 40 $\mathrm{km} / \mathrm{h}$ according to Casapia (2014). One solution to this problem is to build a new road; however, this would require a highly engineered and highly financed process, necessitating the introduction of an alternative means of land transportation for goods such as minerals. As a result, this study addresses in depth the contribution of the railway, whose average speed is $25 \mathrm{~km} / \mathrm{h}$ (with stops at stations and synchronization of locomotives and wagons), with the objective of meeting the requirements of the national productive activity and reduce road congestion produced by it.

\section{Background}

Since its arrival in the country, automotive road transportation has been in competition with the railway, which had been developed 50 years earlier. However, the railroad transportation system in the center and south of the country has been witnessing a slow decline, even as other countries are currently reaching a balanced situation in terms of volumes of cargo being transported.

Peruvian national productivity is strengthened by mining, which is why there has been an expansion of the road network with primacy over rail transport; in this way the Ferrocarril Central (Central Railway) is in direct specific competition with the Carretera Central. According to Casapia (2014), these two modes of competitive transportation comprise the "central bimodal", which connects the regions of Pasco, Junín and Lima with the Port of Callao; therefore, it is deduced that the transportation of freight by trucks is almost four times the freight transported by trains, leading to congestion of the highway (see Figure 1).

1 Civil Engineer from Universidad Ricardo Palma with a specialization in Transportation Infrastructure Concession Regulation (Lima, Peru). Currently working as consultant for Janus Consulting (Cusco, Peru)

ORCID: https://orcid.org/0000-0002-5776-6109

E-mail: janusconsulting.gg@gmail.com 


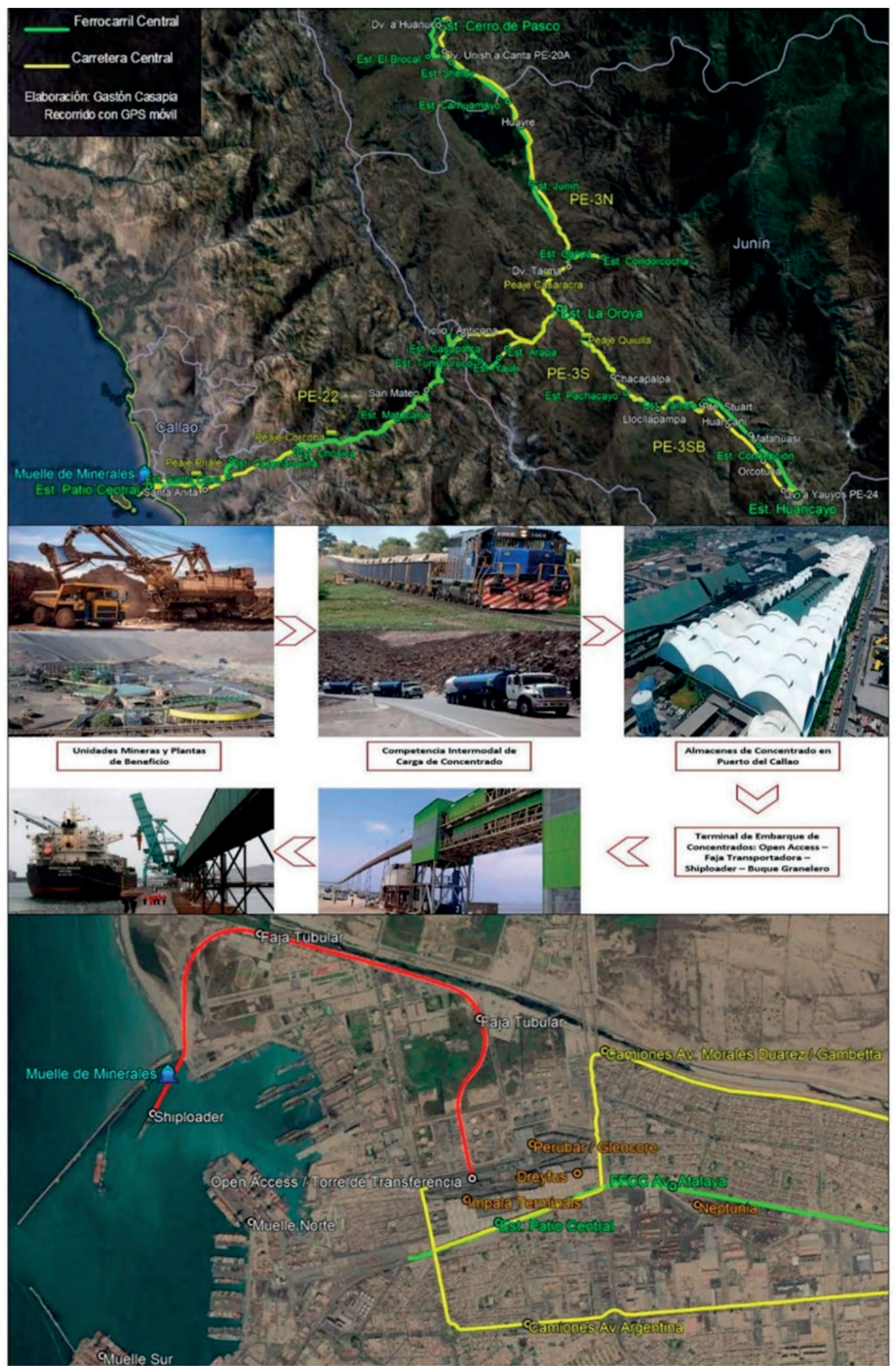

Figure 1. Intermodal competition flow of the "bimodal central" and concentrate loading in Port of Callao.

Source: Prepared by the author with the aid of geospatial tools.

The Carretera Central, with $377 \mathrm{~km}$ of track, has been under concession to Deviandes S.A.C. since 2010, which is charged of performing routine and periodic maintenance in order to postpone its rehabilitation. Its revenues are derived from the collection of tolls in Corcona, Casaracra and Quiulla, to which an additional toll would be added, covered by annual minimum income guaranteed by the Government — that 
for 2016 was USD 14271 590, excluding I.G.V. (General Sales Tax) and for each toll station- as stipulated in the concession agreement of the Supervisory Board for Investment in Public Transport Infrastructure [Ositrán] (2010)

The Ferrocarril Central was concessioned in 1999 to Ferrovías Central Andina S.A., whose infrastructure, tractive stock (locomotives) and rolling stock (coaches and wagons) came from Empresa Nacional de Ferrocarriles (Enafer). The railrway, with a standard gauge of $1.44 \mathrm{~m}$ and a length of $477 \mathrm{~km}$, is being rehabilitated and its rail yard, renovated. Its tariffs are established based on the charge for use of the track per kilometer traveled, from which the Government receives $24.75 \%$ of gross revenues (not including revenues from tractive or rolling stock or I.G.V.) (Ositrán, 2006).

The importance of the storage and shipment of freight in the Central Region extends to the Port of Callao, as it is part of the logistics chain of minerals exported between the mine or concentrating plant and the ships to be loaded at the port. Thus, since the end of 2014, exports have been realized through the concession of the Terminal de Embarque de Concentrados de Minerales to the company Transportadora Callao S.A. which has a hermetic conveyor belt system that goes from a free or public access point (open access) or transfer tower to the dock (shiploader), designed to handle 2000 tonnes per hour and ships of up to 60000 deadweight tonnes (DWT). According to Transportadora Callao (2018). the benefits of this shipping system are that it is 5 times faster than the previous one, it reduces 8 days of waiting time at anchor, it reduces 3 days of dock laytime, and it has replaced approximately 369 000 truck trips between warehouses and the port.

The objective of this research is to analyze the adequate intermodal competition of mining freight transportation to reduce vehicular congestion in the mining logistics chain in the Central Region.

\section{HYPOTHESIS}

Adequate intermodal competition in rail freight transportation reduces vehicular congestion in the mining logistics chain in the Central Region.

\section{JUSTIFICATION}

The traditional mining sector is the main exporter in Peru. In 2017, this sector generated around 61\% of the total value of FOB exports, which amounted to USD 26809000 000, representing an increase of $27.4 \%$ compared with the previous year, due to the increase in exports of copper (36\%), iron (27\%), zinc $(32 \%)$, and other minerals (42\%) (Superintendencia Nacional de Aduanas y de Administración Tributaria [SUNAT], 2018), which has contributed to the increase in GDP, which is the indicator that measures the capacity to generate wealth in the economy of the country.

Due to the absence of refineries in Peru, more than $95 \%$ of mining production, including ore concentrates such as copper, lead, silver, gold, zinc, and molybdenum, is sent to foreign markets. These concentrates have been traded internationally through private companies, which include domestic and foreign producers and traders, who are currently in charge of mobilizing the total exportable production of these concentrates using their logistics and means of transportation.

Due to the extensive use of cargo trucks, with the premise that it is the only means to meet commercial deadlines, year after year the vehicular congestion on the Carretera Central increases at rates for which its transportation infrastructure was not designed.

The Central Region has another much more efficient means of transportation for large volumes of freight and long distances: the Ferrocarril Central. However, it does not have the same equity conditions, even though the Plan Nacional de Desarrollo Ferroviario (National Railway Development Plan) indicates that its purpose is to contribute to the competitiveness of exports (Resolución Ministerial N. ${ }^{\circ}$ 396-2016-MTC, 2016).

Public transportation infrastructure should aim to reach more places and in faster ways, to be more economical, more comfortable and safer, and especially reduce the infrastructure gap and increase the competitiveness of the country. Consequently, this study analyzes the correlation between the problem of the excess of trucks with mining cargo on the Carretera Central, according to intermodal competition, and its mining logistics to ports; thus, the study proposes to reduce its circulation by using the railway, which is more efficient and affordable.

\section{THEORETICAL BASIS}

\section{Intermodal Competition in Freight Transportation}

Intermodal transportation occurs when different means of transportation work together, carrying out the shipment of materials and goods with a single load measure that could be in containers or other transport elements with cargo capacity. 
Taking as a point of reference the modal split of freight transportation in different economies of the world -including 28 countries of the European Union, USA, Japan, China, and Russia-, maritime and road modes move the highest percentage of cargo with $33 \%$ each, while rail transportation is close with $22 \%$ of the total cargo in billion ton-kilometers, according to the European Commission (2018). However, this intermodal competition is far from our reality, where the studies of the Plan Intermodal de Transportes 2004-2023 (Intermodal Transport Plan 2004-2023) establish there is a "intercity cargo" transportation whose $74 \%$ is transported by road, $13 \%$ by rail, $12 \%$ by sea and $1 \%$ by river and air mode (Ministerio de Transportes y Comunicaciones [MTC], 2005).

According to Rodrigue, Comtois and Slack "Los modos pueden competir o complementarse entre sí en términos de costo, velocidad, accesibilidad, frecuencia, seguridad, comodidad, etc. [Modes can compete or complement each other in terms of cost, speed, accessibility, frequency, safety, comfort, etc.]" (2013, p. 107) depending on the following conditions: 1. Different geographic markets, 2. Different transportation markets, and 3. Different levels of service.

While the existence of intermodal competition depends on geographic and transportation markets, there are other factors that affect these principles, such as infrastructure conditions, terrain conditions, and levels of service in collapse or with congestion, so intermodal competition in long-distance freight transportation must be optimized.

According to Thomson and Bull (2002), "la congestión es la condición que prevalece si la introducción de un vehículo en un flujo de tránsito aumenta el tiempo de circulación de los demás [congestion is the condition that prevails if the introduction of one vehicle into a traffic flow increases the circulation time of others]" (p. 110). On this matter, Ortúzar and Willumsen (2011) define that congestion arises when the intensity of demand approaches the capacity of the facility (road or rail infrastructure) and the time required to use it exceeds the established low-demand average.

\section{Mining Marketing Logistics Chain}

The value chain in the mining industry - from the extraction in mines, the processing and the commercialization of concentrates until its export in ports- determines the efficiency of its mining logistics, however, the readjustment of the logistics cost depends on the operators according to their ability to reduce their fixed costs and high rate of logistics specialization, so mining entrepreneurs must decide to select them by communication criteria, freight charge, service capacity, and delivery quality (Galo, Ribeiro, Mergulhão, \& Vieira, 2018).

\section{METHODOLOGY}

This non-experimental study is based on a descriptive-comparative methodological framework. It is a comparative analysis of the demand of land mining cargo transportation on the Carretera Central and the Ferrocarril Central in service in order to know the modal alternative to the existing traffic in vehicular infrastructure.

The modes of land infrastructure for public use in the Central Region are unique, so the population and size of the study are the same; these are the Ferrocarril del Centro and the Carretera IIRSA Centro Tramo 2 (Central IIRSA Highway Section 2).

The mining logistics chain that includes the freight intermodal competition, that is, from the plants in the mining units to the Port of Callao, is evaluated by analyzing (1) the Average Daily Annual Rate (Índice Medio Diario Anual [IMDA]) of the vehicles passing through the counting stations of the Carretera Central and its alternate routes and (2) the demand of trains according to the shipment reports of the Ferrocarril Central. In this way, the number of units arriving to the warehouse at the port is calculated, which then proceed to dispatch the mining cargo by maritime means through the traffic movement of the del Terminal de Embarque de Concentrados de Minerales en el Puerto del Callao (Mineral Concentrate Shipping Terminal at the Port of Callao).

\section{RESULTS}

\section{Mining Productivity in the Central Region}

The area of influence of this study covers the Central Region of the country, in which there has been metal mining production activity for many years, particularly in the Andean terrain of Lima, Junín and Pasco. Therefore, the research is directly circumscribed in 20 mining units, 15 of which have been exploited by underground mining; 4 by surface mining; and 1, Cajamarquilla, by refinery (Ministerio de Energía y Minas [MINEM], 2018) (see Table 1).

\section{Bimodal Central Competition in Mining Freight Transport}

Approximately one century ago, mining freight from the Cerro de Pasco Copper Corporation to La 
Table 1. Mining Units in the Central Region.

\begin{tabular}{|c|c|c|c|c|c|c|c|}
\hline ID & $\begin{array}{l}\text { Expl. } \\
\text { Method }\end{array}$ & Owner & Unit & Reg. & Prov. & Dist. & Prod. \\
\hline 1 & $\begin{array}{l}\text { Undergr. } \\
\text { mining }\end{array}$ & Comp. Minera Argentum S.A. & Anticona & Junín & Yauli & Yauli & $\mathrm{Zn}, \mathrm{Ag}, \mathrm{Cu}, \mathrm{Pb}$ \\
\hline 2 & $\begin{array}{l}\text { Undergr. } \\
\text { mining }\end{array}$ & Comp. Minera Argentum S.A. & Manuelita & Junín & Yauli & Yauli & $\mathrm{Pb}, \mathrm{Zn}, \mathrm{Ag}, \mathrm{Cu}$ \\
\hline 3 & $\begin{array}{l}\text { Undergr. } \\
\text { mining }\end{array}$ & Comp. Minera Argentum S.A. & Morococha & Junín & Yauli & Morococha & $\mathrm{Pb}, \mathrm{Zn}, \mathrm{Ag}, \mathrm{Cu}$ \\
\hline 4 & $\begin{array}{l}\text { Undergr. } \\
\text { mining }\end{array}$ & $\begin{array}{c}\text { Nexa Resources Atacocha } \\
\text { S.A.A. }\end{array}$ & Atacocha & $\begin{array}{l}\text { Pas- } \\
\text { co }\end{array}$ & Pasco & $\begin{array}{l}\text { San Fco. Asís } \\
\text { de Yarusyacán }\end{array}$ & $\begin{array}{c}\mathrm{Pb}, \mathrm{Zn}, \mathrm{Au}, \mathrm{Ag}, \\
\mathrm{Cu}, \mathrm{Bi}\end{array}$ \\
\hline 5 & $\begin{array}{l}\text { Undergr. } \\
\text { mining }\end{array}$ & Comp. Minera Casapalca S.A. & Americana & Junín & Yauli & Yauli & $\mathrm{Zn}, \mathrm{Ag}, \mathrm{Cu}, \mathrm{Pb}$ \\
\hline 6 & $\begin{array}{l}\text { Undergr. } \\
\text { mining }\end{array}$ & $\begin{array}{c}\text { Comp. Minera San Ignacio de } \\
\text { Morococha S.A.A. }\end{array}$ & Palmapata & Junín & Chancha-mayo & San Ramón & $\mathrm{Pb}, \mathrm{Zn}$ \\
\hline 7 & $\begin{array}{l}\text { Undergr. } \\
\text { mining }\end{array}$ & $\begin{array}{l}\text { Comp. Minera San Ignacio de } \\
\text { Morococha S.A.A. }\end{array}$ & San Vicente & Junín & Chancha-mayo & Vitoc & $\mathrm{Pb}, \mathrm{Zn}$ \\
\hline 8 & $\begin{array}{l}\text { Surface } \\
\text { mining }\end{array}$ & $\begin{array}{l}\text { Emp. Administradora Cerro } \\
\text { S.A.C. }\end{array}$ & Cerro de Pasco & $\begin{array}{l}\text { Pas- } \\
\text { co }\end{array}$ & Pasco & Simón Bolívar & $\begin{array}{c}\mathrm{Cu}, \mathrm{Pb}, \mathrm{Zn}, \mathrm{Au}, \\
\mathrm{Ag}\end{array}$ \\
\hline 9 & $\begin{array}{l}\text { Undergr. } \\
\text { mining }\end{array}$ & $\begin{array}{c}\text { Emp. Minera Los Quenuales } \\
\text { S.A. }\end{array}$ & Yauliyacu & Lima & Huarochirí & Chicla & $\mathrm{Zn}, \mathrm{Ag}, \mathrm{Pb}, \mathrm{Cu}$ \\
\hline 10 & $\begin{array}{l}\text { Undergr. } \\
\text { mining }\end{array}$ & $\begin{array}{c}\text { Nexa Resources El Porvenir } \\
\text { S.A.C. }\end{array}$ & Milpo №1 & $\begin{array}{l}\text { Pas- } \\
\text { co }\end{array}$ & Pasco & Yanacancha & $\begin{array}{c}\mathrm{Cu}, \mathrm{Pb}, \mathrm{Zn}, \mathrm{Au}, \\
\mathrm{Ag}\end{array}$ \\
\hline 11 & $\begin{array}{l}\text { Surface } \\
\text { mining }\end{array}$ & Minera Chinalco Perú S.A. & Toromocho & Junín & Yauli & Morococha & $\begin{array}{c}\mathrm{Cu}, \mathrm{Mo}, \mathrm{Zn}, \mathrm{Ag}, \\
\text { As }\end{array}$ \\
\hline 12 & $\begin{array}{l}\text { Undergr. } \\
\text { mining }\end{array}$ & $\begin{array}{l}\text { Soc. Minera Austria Duvaz } \\
\text { S.A.C. }\end{array}$ & Austria Duvaz & Junín & Yauli & Morococha & $\mathrm{Pb}, \mathrm{Zn}, \mathrm{Ag}, \mathrm{Cu}$ \\
\hline 13 & $\begin{array}{l}\text { Surface } \\
\text { mining }\end{array}$ & Soc. Minera El Brocal S.A.A. & Colquijirca Nº1 & $\begin{array}{l}\text { Pas- } \\
\text { co }\end{array}$ & Pasco & Simón Bolívar & $\mathrm{Cu}, \mathrm{Au}, \mathrm{Ag}, \mathrm{As}$ \\
\hline 14 & $\begin{array}{l}\text { Surface } \\
\text { mining }\end{array}$ & Soc. Minera El Brocal S.A.A. & Colquijirca $\mathrm{N}^{\circ} 2$ & $\begin{array}{l}\text { Pas- } \\
\text { co }\end{array}$ & Pasco & Tinyahuarco & $\mathrm{Zn}, \mathrm{Ag}, \mathrm{Pb}, \mathrm{Cu}$ \\
\hline 15 & $\begin{array}{l}\text { Undergr. } \\
\text { mining }\end{array}$ & Volcan Comp. Minera S.A.A. & Andaychagua & Junín & Yauli & Huay-Huay & $\mathrm{Zn}, \mathrm{Ag}, \mathrm{Pb}, \mathrm{Cu}$ \\
\hline 16 & $\begin{array}{l}\text { Undergr. } \\
\text { mining }\end{array}$ & Volcan Comp. Minera S.A.A. & Carahuacra & Junín & Yauli & Yauli & $\mathrm{Zn}, \mathrm{Ag}, \mathrm{Cu}, \mathrm{Pb}$ \\
\hline 17 & $\begin{array}{l}\text { Undergr. } \\
\text { mining }\end{array}$ & Volcan Comp. Minera S.A.A. & Morada & Junín & Yauli & Yauli & $\mathrm{Cu}, \mathrm{Pb}, \mathrm{Zn}, \mathrm{Ag}$ \\
\hline 18 & $\begin{array}{l}\text { Undergr. } \\
\text { mining }\end{array}$ & Volcan Comp. Minera S.A.A. & San Cristóbal & Junín & Yauli & Yauli & $\mathrm{Cu}, \mathrm{Pb}, \mathrm{Zn}, \mathrm{Ag}$ \\
\hline 19 & $\begin{array}{l}\text { Undergr. } \\
\text { mining }\end{array}$ & Volcan Comp. Minera S.A.A. & Ticlio & Junín & Yauli & Morococha & $\mathrm{Cu}, \mathrm{Pb}, \mathrm{Zn}, \mathrm{Ag}$ \\
\hline 20 & Refinery & $\begin{array}{l}\text { Nexa Resources Cajamarqui- } \\
\text { Ila S.A. }\end{array}$ & $\begin{array}{l}\text { Refinería de } \\
\text { Cajamarquilla }\end{array}$ & Lima & Lima & Lurigancho & $\mathrm{Cd}, \mathrm{Cu}, \mathrm{Zn}, \mathrm{Ag}$ \\
\hline
\end{tabular}

Source: Prepared by the author based on the Mapa de Principales Unidades Mineras en Producción (MINEM, 2018).

Oroya and El Callao was transported by railway. However, the later appearance of heavy goods vehicles and the completion of the Carretera Central in 1936 showed that its logistics could be reduced, especially because there was neither at the time nor at the present day a fair price between the toll value per axle and the equivalent deterioration per axle caused by overloading the road.

Since it is difficult to obtain information on the transportation of minerals through the country's highways, a count of land transportation units is made when these vehicles cross checkpoints or stations. In this way the Ministry of Transportation and Com- munications obtains the IMDA by type of vehicle per road sections. In the case of the Central Region, data from the MTC (MTC, 2013 and MTC, 2016) are taken into account —considering that 2016 was the last year in which MTC published this informationand Table 2 and Figure 2 are analyzed.

Table 2 shows the number of vehicles using the Carretera Central (PE-22, PE-3N and PE-3S) and the alternate routes in both directions. According to the classification by demand of the Manual de Carreteras of the MTC (Resolución Directoral N 03-2018MTC, 2018), a road with an IMDA greater than 6000 veh/day, with two or more lanes of $3.60 \mathrm{~m}$ minimum 
Table 2. IMDA 2013 - 2016 in the Central Region.

\begin{tabular}{|c|c|c|c|c|c|c|c|c|c|c|c|}
\hline \multirow{2}{*}{ Control Station } & \multirow{2}{*}{$\begin{array}{c}\text { Route } \\
\text { (both ways) }\end{array}$} & \multicolumn{5}{|c|}{2013} & \multicolumn{5}{|c|}{2016} \\
\hline & & L.V. & P.T. & F.T. & IMDA & T3S3 & L.V. & P.T. & F.T. & IMDA & T3S3 \\
\hline \multirow{2}{*}{ Corcona } & \multirow{2}{*}{ PE-22 } & 2150 & 1034 & 2376 & 5560 & 1061 & 2570 & 1090 & 2539 & 6199 & 1077 \\
\hline & & $39 \%$ & $19 \%$ & $43 \%$ & & $45 \%$ & $41 \%$ & $18 \%$ & $41 \%$ & & $42 \%$ \\
\hline \multirow{2}{*}{ Tambo de Viso } & \multirow{2}{*}{ PE-22 } & 2121 & 750 & 2558 & 5429 & 1081 & 2370 & 876 & 2764 & 6010 & 1182 \\
\hline & & $39 \%$ & $14 \%$ & $47 \%$ & & $42 \%$ & $39 \%$ & $15 \%$ & $46 \%$ & & $43 \%$ \\
\hline \multirow{2}{*}{ Casaracra } & \multirow{2}{*}{ PE-3N } & 1272 & 446 & 1730 & 3448 & 688 & 1776 & 654 & 2112 & 4542 & 795 \\
\hline & & $37 \%$ & $13 \%$ & $50 \%$ & & $40 \%$ & $39 \%$ & $14 \%$ & $46 \%$ & & $38 \%$ \\
\hline \multirow{2}{*}{ Carhuamayo } & \multirow{2}{*}{ PE-3N } & 1054 & 302 & 1017 & 2373 & 490 & 1223 & 407 & 1717 & 3347 & 788 \\
\hline & & $44 \%$ & $13 \%$ & $43 \%$ & & $48 \%$ & $37 \%$ & $12 \%$ & $51 \%$ & & $46 \%$ \\
\hline \multirow{2}{*}{ Quiulla } & \multirow{2}{*}{ PE-3S } & 1520 & 517 & 884 & 2921 & 464 & 2723 & 617 & 984 & 4324 & 352 \\
\hline & & $52 \%$ & $18 \%$ & $30 \%$ & & $52 \%$ & $63 \%$ & $14 \%$ & $23 \%$ & & $36 \%$ \\
\hline \multirow{2}{*}{ Churín } & \multirow{2}{*}{ PE-18 } & 219 & 63 & 130 & 412 & 35 & 585 & 196 & 245 & 1026 & 80 \\
\hline & & $7 \%$ & $2 \%$ & $4 \%$ & & $27 \%$ & $14 \%$ & $5 \%$ & $6 \%$ & & $33 \%$ \\
\hline \multirow{2}{*}{ Yangas } & \multirow{2}{*}{ PE-20A } & 480 & 209 & 66 & 755 & 8 & 737 & 306 & 173 & 1216 & 34 \\
\hline & & $16 \%$ & $7 \%$ & $2 \%$ & & $12 \%$ & $17 \%$ & $7 \%$ & $4 \%$ & & $20 \%$ \\
\hline \multirow{2}{*}{ Pirca } & \multirow{2}{*}{ PE-1NC } & 217 & 35 & 26 & 278 & 0 & 282 & 50 & 32 & 364 & 0 \\
\hline & & $7 \%$ & $1 \%$ & $1 \%$ & & $0 \%$ & $7 \%$ & $1 \%$ & $1 \%$ & & $0 \%$ \\
\hline \multirow{2}{*}{ Pacarán } & \multirow{2}{*}{ PE-24 } & 220 & 141 & 67 & 428 & 0 & 324 & 235 & 80 & 639 & 2 \\
\hline & & $8 \%$ & $5 \%$ & $2 \%$ & & $0 \%$ & $7 \%$ & $5 \%$ & $2 \%$ & & $3 \%$ \\
\hline
\end{tabular}

Note: L.V.: Light Vehicle (cars and vans).

P.T.: Public Transportation (rural vans, buses and omnibuses).

F.T.: Freight Transportation (articulated , 2 and 3 axles trucks).

Source: Prepared by the author based on IMDA by type of vehicle and road sections (MTC, 2013; MTC, 2016).

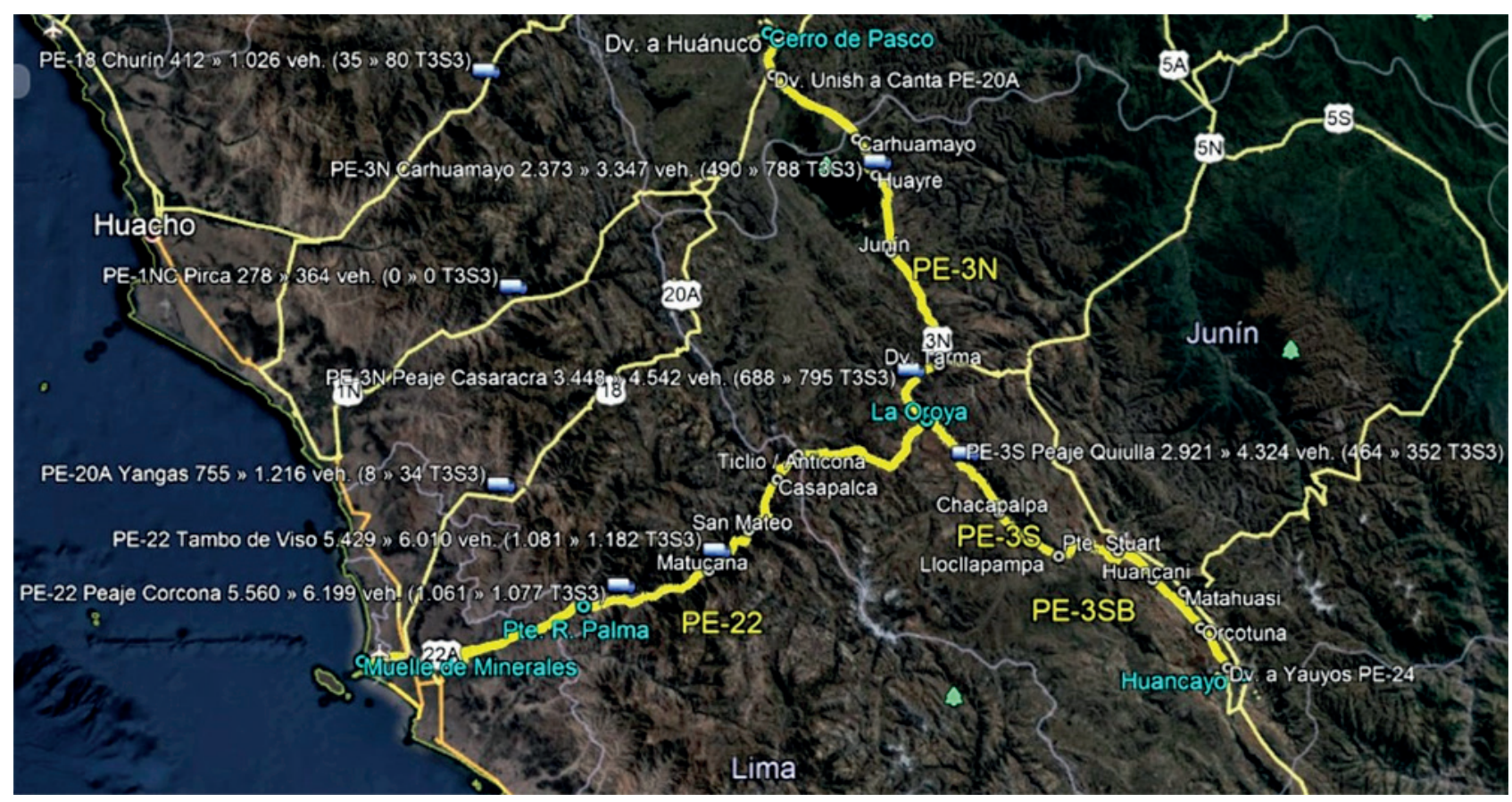

Figure 2. IMDA 2013 - 2016 in the Central Region.

Source: Prepared by the author on Google Earth, based on IMDA, and road sections (MTC, 2013; MTC, 2016). 
width and a central separator of $6.00 \mathrm{~m}$ would be an autopista de primera clase (first class highway); that means that its typical section would measure a minimum width of $20.40 \mathrm{~m}$. However, the Carretera Central still has a $6.60 \mathrm{~m}$ roadway, which is why it still presents inconveniences with its geometric design and, therefore, vehicular congestion problems.

It is worth noting that the flow of six-axle heavy goods vehicles, such as T3S3 trucks (three-axle tractor and three-axle semitrailer) and the box trucks used to transport concentrates to the port, has been increasing at an average annual rate of 1 to $5 \%$. However, in Carhuamayo (between Cerro de Pasco and La Oroya) this increase was as high as $20 \%$, while on other routes such as PE-18 in Churin (between Huaura and Ambo) the increase was 43\% and on PE-20A in Yangas (between Lima and Unish) it was even $108 \%$ per year.

In addition to analyzing the transfer of mineral concentrates in heavy goods vehicles, it is also possible to compare the movement of trains (locomotives and wagons) and their respective loads through the Ferrocarril Central, whose information comes from the concessionaire Ferrovías Central Andina S.A.
(FVCA), which is shown in Table 3, regarding the transport of mineral concentrates (Ferrovías Central Andina [FVCA], 2017).

Once the zinc concentrate arrives in Callao, a total of 10037 WMT were transferred to the Refinería de Cajamarquilla by Nexa Resources Cajamarquilla S.A. to obtain zinc bars.

In 2017, the transport of copper, lead and zinc concentrate through the railroad line to the Port of Callao (Central Yard) initiated in the beneficiation plants and/or mining units in production. The main customers of the railroad, according to concentrate, were Minera Chinalco with $99 \%$ of copper; Volcan with $92 \%$ of zinc; and, in the case of lead, Milpo with $51 \%$ and Atacocha with $46 \%$; the latter two have now become part of the company Nexa Resources, which would consequently take $97 \%$ of the lead.

\section{Mining Logistics at the Port of Callao}

Regardless of the supply chain to the mines, the logistics chain organizes the transportation to deliver the products from the beneficiation plants to the final customer. Whether using the bimodal central

Table 3. Freight Transport in Ferrocarril Central to Callao in 2017.

\begin{tabular}{|c|c|c|c|c|}
\hline \multicolumn{5}{|c|}{ Cu concentrate from M.U. /plant to the Port of Callao } \\
\hline Company & Origin & Destination & WMT & $\%$ \\
\hline Minera Chinalco Perú S.A. & Tunshuruco & Patio Central & 1169924 & $99 \%$ \\
\hline Sociedad Minera El Brocal S.A.A. & El Brocal & Patio Central & 11374 & $1 \%$ \\
\hline Milpo Andina Perú S.A.C. & Unish & Patio Central & 1264 & $0 \%$ \\
\hline Compañía Minera Atacocha S.A.A. & Unish & Patio Central & 158 & $0 \%$ \\
\hline & & & 1182719 & \\
\hline \multicolumn{5}{|c|}{$\mathrm{Pb}$ concentrate from M.U. /plant to the Port of Callao } \\
\hline Company & Origin & Destination & WMT & $\%$ \\
\hline Milpo Andina Perú S.A.C. & Unish & Patio Central & 15443 & $51 \%$ \\
\hline Compañía Minera Atacocha S.A.A. & Unish & Patio Central & 14108 & $46 \%$ \\
\hline Volcan Compañía Minera S.A.A. & Mahr Túnel & Patio Central & 835 & $3 \%$ \\
\hline & & & 30385 & \\
\hline \multicolumn{5}{|c|}{ Zn concentrate from M.U. /plant to the Port of Callao } \\
\hline Company & Origin & Destination & WMT & $\%$ \\
\hline Volcan Compañía Minera S.A.A. & Carahuacra / Mahr Túnel & Patio Central & 173562 & $92 \%$ \\
\hline Milpo Andina Perú S.A.C. & Unish & Patio Central & 9115 & $5 \%$ \\
\hline Sociedad Minera El Brocal S.A.A. & El Brocal & Patio Central & 4131 & $2 \%$ \\
\hline Empresa Administradora Cerro S.A.C. & Cerro de Pasco & Patio Central & 1607 & $1 \%$ \\
\hline Empresa Minera Los Quenuales S.A. & Casapalca & Patio Central & 161 & $0 \%$ \\
\hline & & & 188577 & \\
\hline
\end{tabular}

Source: Prepared by the author based on the Reporte Acumulado de Clientes por Carga (FVCA, 2017). 
or other alternative routes, the purpose of the mining companies is to complete the supply chain to a refinery or to the Port of Callao, depending on the commercialization contract they have signed.

Due to the requirements with respect to mineral deliveries, most mining companies subcontract the organization of transportation to logistics operators or traders, which offer mining logistics consulting services, which consist of contracting transportation companies, picking up and moving the goods, contracting warehouses for storage, dealing with customs procedures and maritime shipments, etc. Trafigura Perú S.A.C. (formerly Cormín) and Glencore Perú S.A.C. are two examples of these logistics operators.

The Plan de Negocios del Terminal de Embarque de Concentrados (Transportadora Callao, 2018) details the 2017 traffic of the feeder warehouses used in port, such as Impala Terminals with 45\%, Perubar with 33\%, Chinalco with 15\%, and Dreyfus with $7 \%$. This is complemented by the information from the Resumen General de Naves y Tráfico de Carga en el Terminal de Embarque de Concentrados de Minerales (Ositrán, 2018), which is shown in Table 4.
In 2017, 182 bulk carriers with an average cargo of $16336 \mathrm{t}$ per vehicle, that gave a total of 2973090 tonnes, were served. This represented $77 \%$ of the total ore concentrates exported in the Port of Callao and $26 \%$ of the total exported in Peru.

The main problem at the Port of Callao is that part of the concentrates is still exported in big bags inside containers, which is equivalent to $23 \%$ of total exports. For this reason, the concessionaire has been registering losses in its income statement, since the cargo handled by the Terminal is substantially less than projected.

Based on the information collected on the mining units in the Central Region and their concentrating plants, it is possible to estimate the number of heavy goods trucks that travel through this region, the number of wagons transporting ore concentrate and the exports that make use of the conveyor belt of the Terminal de Embarque de Concentrados de Minerales del Callao.

\section{DISCUSSION}

In 2017, Transportadora Callao mobilized a total of 2973090 WMT of copper, zinc and lead concentrates. If it is considered that in the same year

Table 4. Export of Concentrates from Transportadora Callao 2017.

\begin{tabular}{|c|c|c|c|c|c|}
\hline Month & $\begin{array}{c}\text { Total } \\
\text { Bulk } \\
\text { Carriers } \\
\end{array}$ & $\begin{array}{l}\text { Solid Bulk - Mineral Zn } \\
\text { (t) }\end{array}$ & $\begin{array}{l}\text { Solid Bulk - Mineral Cu } \\
\text { (t) }\end{array}$ & $\begin{array}{l}\text { Solid Bulk - Mineral Pb } \\
\text { (t) }\end{array}$ & $\begin{array}{l}\text { Total Exports } \\
\text { (t) }\end{array}$ \\
\hline January & 17 & 79596 & 132853 & & 212449 \\
\hline February & 18 & 98092 & 163638 & 48239 & 309968 \\
\hline March & 14 & 90545 & 81275 & 14044 & 185865 \\
\hline April & 12 & 60495 & 133774 & 19448 & 213718 \\
\hline May & 14 & 138558 & 146879 & 25241 & 310678 \\
\hline June & 20 & 117725 & 123643 & 33404 & 274772 \\
\hline July & 12 & 80737 & 85057 & 29781 & 195576 \\
\hline August & 11 & 54873 & 116683 & 14937 & 186494 \\
\hline September & 17 & 126418 & 190910 & 16324 & 333652 \\
\hline October & 13 & 82877 & 92490 & 29045 & 204412 \\
\hline November & 16 & 104787 & 127109 & 19495 & 251391 \\
\hline December & 18 & 141159 & 112332 & 40625 & 294116 \\
\hline \multirow[t]{2}{*}{ Annual } & 182 & 1175862 & 1506644 & 290584 & 2973090 \\
\hline & & $40 \%$ & $51 \%$ & $10 \%$ & $100 \%$ \\
\hline \multicolumn{4}{|c|}{ Total WMT exported by Transportadora Callao } & 2973090 & $77 \%$ \\
\hline \multicolumn{4}{|c|}{ Total WMT exported in big bags in containers } & 880385 & $23 \%$ \\
\hline \multicolumn{4}{|c|}{ Total WMT exported by the Port of Callao } & 3853475 & \\
\hline
\end{tabular}

Source: Prepared by the author based on the Resumen General de Naves y Tráfico de Carga en Terminal de Embarque de Concentrados de Minerales (Ositrán, 2018). 
the Ferrocarril Central transported to the port 1391 644 WMT of concentrates with the same characteristics, we can deduce that the difference in these amounts are the tonnes that were sent by trucks to Callao, that is, 1581446 WMT. This means that $47 \%$ was mobilized by railway wagons and $53 \%$ by trucks.

Since there is no further research on this subject, except for the comparative of the bimodal central, and based on the above, it is argued that most mining companies subcontract to traders who prefer to use trucking companies.

Considering that a box truck of mining concentrate has an average payload of 30 WMT, the number of trucks per year that arrived at the port was 52715 , with a frequency of 144 trucks per day.

\section{HYPOTHESIS TESTING}

As it is shown in Table 2, the IMDA at Corcona control station was 6199 vehicles. If the cargo is transferred from road to rail, removing only an average of 288 trucks in both directions, the IMDA would be reduced by almost $5 \%$. If the trucks transporting big bags, as shown in Table 4, are included in the cargo shifting, about 448 units would be replaced per day, that is, $42 \%$ of 6 -axle trucks. As a result, a decongestion of heavy vehicles would occur not only on the highway but particularly in the access to Lima and the Port of Callao. Thus, the analysis shows that adequate intermodal competition of rail freight transportation reduces vehicle congestion in the mining logistics chain.

\section{CONCLUSIONS AND RECOMENDATIONS}

The development of the infrastructure of land transportation has been consolidating according to the technological prominence of the means of transportation. Thus, the average operating costs of a truck -fuel, maintenance, spare parts and insuranceare significantly lower than the tractive and rolling stock used by a train. Likewise, road transport infrastructure with a flexible pavement has a lower investment cost than railways (Casapia, 2014); however, road transport receives a higher subsidy, despite the fact that railroads have higher sunk costs, with an average use period of 80 years in operation before being rehabilitated.

After the discussion, it is clear that the railroad mode might guarantee the socioeconomic benefit of the country, reducing vehicle congestion, saving travel time and vehicle operation costs, decreasing traffic accidents, and above all reducing the costs derived from the intensive use of trucks. In other words, a policy of safety, environmental health and social responsibility should take precedence.

The improvement of transportation infrastructure is necessary to reduce logistics costs and to increase the sustainable growth of the region in order to close the infrastructure gap that in our country would be a good option to achieve the strength of the Plan Nacional de Infraestructura para la Competitividad (D. S. N. $\left.{ }^{\circ} 238-2019-E F, 2019\right)$, whose short-term gap in rail transport considers S/20 430000 000, through multimodal connectivity, integrating productivity and its regions using road, rail and water transportation modes.

\section{REFERENCES}

[1] Casapia, G. (2014). Carretera o Ferrocarril ¿Qué modo de transporte usar? Lima, Peru: Universidad Ricardo Palma.

[2] D. S. N. ${ }^{\circ}$ 238-2019-EF. Plan Nacional de Infraestructura para la Competitividad. Diario Oficial El Peruano (July 28, 2019).

[3] European Commission. (2018). EU Transport Statistical Pocketbook. Luxembourg, Publications Office of the European Union. Retrieved from https://doi.org/10.2832/491038

[4] Ferrovías Central Andina - FVCA (2017). Reporte Acumulado de Clientes por Carga. Lima, Peru.

[5] Galo, N., Ribeiro, P., Mergulhão, R., \& Vieira, J. (2018). Selección de proveedor de servicios logísticos: alineación entre criterios e indicadores. Innovar, 28(69), 55-67. Retrieved from https://doi.org/10.15446/innovar. v28n69.71696

[6] Ministerio de Energía y Minas. (2018). Mapa de las Principales Unidades Mineras en Producción 2018. Lima, Peru.

[7] Ministerio de Transportes y Comunicaciones. (2005). Plan Intermodal de Transportes PIT 2004-2023. Lima, Peru.

[8] Ministerio de Transportes y Comunicaciones. (2013). Índice Medio Diario Anual - IMDA. Lima, Perú.

[9] Ministerio de Transportes y Comunicaciones (2016). Índice Medio Diario Anual - IMDA. Lima, Perú. 
[10] Organismo Supervisor de la Inversión en Infraestructura de Transporte de Uso Público. (2006). Contrato de Concesión del Ferrocarril del Centro. Lima, Peru.

[11] Organismo Supervisor de la Inversión en Infraestructura de Transporte de Uso Público. (2010). Contrato de Concesión del IIRSA Centro Tramo 2: Puente Ricardo Palma - La Oroya - Huancayo y La Oroya - Dv. Cerro de Pasco. Lima, Peru.

[12] Organismo Supervisor de la Inversión en Infraestructura de Transporte de Uso Público. (2018). Resumen General de Naves y Tráfico de Carga en el Terminal de Embarque de Concentrados de Minerales 2017. Lima, Peru.

[13] Ortúzar, J., \& Willumsen, L. (2011). Modelling Transport. Chichester, UK: Wiley Publication. Retrieved from https://doi. org/10.1002/9781119993308

[14] Resolución Ministerial N. ${ }^{\circ}$ 396-2016-MTC. Aprueban el "Plan Nacional de Desarrollo Ferroviario". Diario Oficial El Peruano (June 16, 2016).
[15] Resolución Directoral N N 03-2018-MTC, Aprueban Manual de Carreteras: Diseño Geométrico DG 2018. Diario Oficial El Peruano (February 7, 2018).

[16] Rodrigue, J., Comtois, C., \& Slack, B. (2013). The Geography of Transport Systems. Abingdon, UK: Routledge, Taylor \& Francis Group. Retrieved from https://doi. org/10.4324/9780203371183

[17] Superintendencia Nacional de Aduanas y de Administración Tributaria (2018). Anuario Estadístico de Comercio Exterior 2017. Lima, Peru.

[18] Thomson, I., \& Bull, A. (2002). La Congestión del Tránsito Urbano: Causas y Consecuencias Económicas y Sociales. Revista de la CEPAL, (76), 109-121. Retrieved from http://hdl.handle. net/11362/10804

[19] Transportadora Callao (2018). Plan de Negocios del Terminal de Embarque de Concentrados de Minerales. Lima, Peru. 